(Aus dem physiologischen Institut der Universität Kiel.)

\title{
Eine Methode, die elektrische Leitfähigkeit im Innern von Zellen zu messen.
}

Von

\section{Rudolf Höber.}

\author{
(Mit 7 Textfiguren.)
}

\begin{abstract}
Über die elektrische Leitfähigkeit des Inhalts von Zellen ist bisher nichts bekannt; dies heisst zugleich: wir kennen noch nicht den Zustand der Salze im Innern der Zellen. Misst man den elektrischen Widerstand von Geweben nach dem üblichen Verfahren von Kohlrausch mit Brücke und Telephon, so findet man, dass die Zellen, solange sie leben, dem elektrischen Strom einen grossen Widerstand bieten, während nach dem Absterben der Widerstand stark sinkt. Die elektrische Leitfähigkeit des Blutes rührt nach den bekannten Untersuchungen von S te wart, B ugarszky und Tangl, Roth und Oker-Blom so gut wie ganz vom Plasma her, die durch Zentrifugieren aus dem Plasma herausgeschleuderten, aber auf diese Weise keineswegs völlig vom Plasma befreiten Blutkörperchen leiten daher den Strom mehr als 100 mal schlechter als das Plasma; zerstört man nun die Blutkörperchen, etwa mit Saponin (Stewart), so schnellt die Leitfähigkeit in wenigen Minuten in dem Maass in die. Höhe, als wäre die Elektrolytkonzentration auf das 40 - bis 50 fache gestiegen. Dies ebenso wie die Widerstandsabnahme beim Absterben eines jeden Gewebes kann entweder darauf beruhen, dass die normale Oberfläche der Zellen eine dielektrische Hülle um einen elektrolytischen Inhalt darstellt, und dass im Tode die isolierende. Eigenschaft der Hülle verloren geht, oder darauf, dass dureh eine Absterbereaktion vorher gebundene Elektrolyte im Innern frei werden. Welche Erklärung die richtige ist, liess sich bisher nur auf indirektem Wege und nur unsicher entscheiden. Inwiefern eine sichere Entscheidung von Interesse wäre, wird später erörtert werden. Zu-
\end{abstract}


nächst soll eine Methode beschrieben werden, mit der es glückt, die „innere Leitfähigkeit" der Zellen direkt zu messen.

Prinzip der Methode: Wenn man zwischen die Platten eines Kondensators von der Kapazität

$$
C=\varepsilon \frac{F l}{4 \pi d} \cdot \text {. . . . . }
$$

wo $F l$ die Fläche der Kondensatorplatten, $d$ ihren Abstand und $\varepsilon$ die Dielektrizitätskonstante bedeutet, eine starke Metallplatte von per Fläche $F l$ und der Dicke $d_{3}$ schiebt (Fig. 1), so vergrössert sich

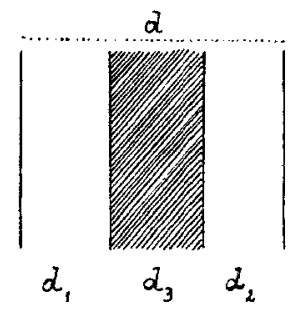

Fig. 1. die Kapazität; sie wird so gross, wie wenn zwei Kondensatoren von der Fläche $F l$ und den Abständen $d_{1}$ und $d_{2}$ hintereinandergeschaltet, oder wie wenn die Kondensatorplatten aus dem Abstand $d$ um $d_{3}$ einander genähert wären. Die Kapazität beträgt nun:

$$
C_{1}=\varepsilon \frac{F l}{4 \pi\left(d_{1}+d_{2}\right)} \cdot \text {. . . . . }
$$

Nicht grundsätzlich hiervon verschieden ist folgender Fall: in einem parallelepipedischen Trog, welcher mit einer Mischung aus Chloroform und Benzol vom spezifischen Gewicht 1 gefüllt ist, tauchen zwei Metallplatten; der Trog hat dann eine durch die Gleichung 1 gegebene Kapazität. Giesst man nun etwas Wasser in den Trog und stellt durch Umrühren eine Emulsion her, so steigt die Kapazität um so mehr, je mehr Wasser zugesetzt ist. Die Erklärung ist darin zu suchen, dass Wasser gegenüber dem Gemisch aus Chloroform. und Benzol als unendlich guter Leiter fungiert, es ist also in seiner Wirkung der starken Metallplatte vergleichbar, welche vorher, zwischen die Kondensatorplatten geschoben, kapazitätserhöhend wirkte. Der eben geschilderte Versuch ist vor längerer Zeit von Millikan ${ }^{1}$ ) auf Anregung von Nernst ausgefuhrt worden, er schien mir das Prinzip für eine Messung der inneren Leitfähigkeit der Zellen zu enthalten. Denn denkt man sich an Stelle der Wassertröpfehen der Emulsion Blutkörperchen, an Stelle der ChloroformBenzolmischung eine mit den Blutkörperchen isotonische, für sie indifferente reine wässerige Nichtleiterlösung, so müssen für den Fall, dass die Elektrolyte im Innern der Blutkörperchen frei sind, diese letzteren sich gegenüber der Nichtleiterlösung als sehr gute Leiter 
verhalten und dementsprechend kapazitätssteigernd wirken. Es handelte sich also darum, Kapazitätsmessungen an wässerigen Lösungen vorzunehmen. An und für sich bieten nun entsprechende Kapazitätsmessungen keine Schwierigkeiten, sie können bequem in einer von einem Wechselstrom gespeisten Wheatstone'schen Brücke vorgenommen werden, in der zwei Widerstände durch zwei Kapazitäten ersetzt sind. Sind in der Fig. $2 O_{1}$ und $C_{2}$ zwei Kapazitäten, $w_{1}$ und $w_{2}$ zwei Widerstände, $T$ ein Telephon als Nullinstrument, so verhält sich, wenn durch die Brücke kein Strom fliesst,

$$
w_{1}: w_{2}=C_{2}: C_{1} \cdot \text {. (3). }
$$

Wie aber vor allem $\mathrm{Nernst}^{1}$ ) gelegentlich seiner dielektrischen Messungen gezeigt hat, kommen bei Verwendung von Wasser oder wässerigen Lösungen als Dielektrikum Störungen vor, hauptsächlich dadurch, dass Wasser nicht ganz unbeträchtlich den elek-

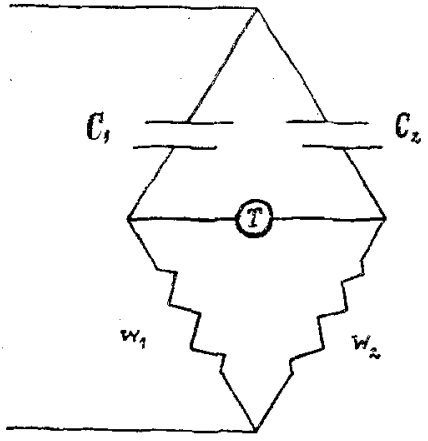

Fig. 2. trischen Strom leitet, und dass sich infolgedessen bei Verwendung der gewöhnlichen niedrigfrequenten Wechselströme Polarisationskapazitäten ausbilden. Über diese Schwierigkeiten kann man jedoch nach Nernst durch Verwendung höherfrequenter Wechselströme, namentlich durch Verwendung von Schwingungen, wegkommen.

Versuchsanordnung: Für die spezielle Ausarbeitung der Methode der Messung der inneren Leitfähigkeit habe ich nun versucht, mir die ausserordentlich reiche Erfahrung von Nerust im Gebiet der dielektrischen Messungen zu Nutze zu machen, und ihn um seinen Rat gebeten. Geheimrat Nernst ist mit dem grössten Entgegenkommen auf meinen Plan eingegangen, er hat mir das gleich zu beschreibende Verfahren zu dessen Ausführung empfohlen und mir während einiger physikalischer Vorversuche, die ich in seinem Institut anstellte, ununterbrochen seine Hilfe gewährt. Ich sage ihm auch an dieser Stelle meinen wärmsten Dank dafür. -

Es wurde soeben gezeigt, dass man feststellen kann, ob Zellen eine erhebliche innere Leitfähigkeit besitzen oder nicht, indem man zusieht, ob die Kapazität eines Troges durch Eintragen der Zellen

1) Nernst, Wiedemann's Annalen Bd. 60 S. 600 (1897) und Zeitschr. f. physik. Chemie Bd. 14 S. 622 (1894). 
in sein wässeriges Dielektrikum geändert wird, oder ob das nicht geschieht. Will man aber das Verfahren zu einer quantitativen Messung der inneren Leitfähigkeit ausbilden, dann ist zuvor zu entscheiden, ob die Methode überhaupt verschiedene Grade der inneren Leitfähigkeit erkennen lässt. $\mathrm{Zu}$ diesem $\mathrm{Zweck}$ wurden die Blutkörperchen zunächst sozusagen imitiert durch Glasröhrchen, welche mit verschieden konzentrierten Elektrolytlösungen gefüllt wurden; diese Röhrchen wurden in einen parallelepipedischen Trog gehängt, der mit Wasser gefüllt und mit Silberplatten als Elektroden versehen war. Fig. 3 gibt ein Bild des Troges.

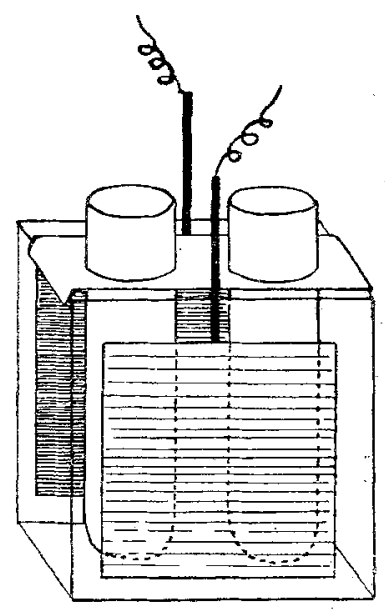

Fig. 3.

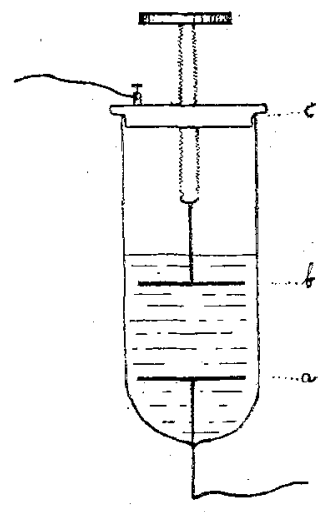

Fig. 4.

Die Kapazität dieses Troges wurde nun bei verschiedener Füllung der Röbrchen mit einem zweiten „Messtrog" (Fig. 4) verglichen. Dieser bestand aus einem zylindrischen Gefäss, in welches senkrecht zu seiner Achse zwei kreisrunde Silberelektroden eingesetzt waren, die eine (a) fest, die andere (b) mit Hilfe einer durch den Hartgummideckel $c$ geführte Mikrometerschraube parallel zur ersten versehieblich. Der Trog wurde mit Wasser gefüllt. Kapazitätsänderungen des Röhrchentroges konnten so durch Verschiebung der Elektroden am Messtrog kompensiert und durch den jeweiligen Abstand der Elektroden zahlenmässig gemessen werden.

Die beiden Tröge wurden nun in folgende Brückenanordnung eingesehaltet $^{1}$ ) (Fig. 5): $I$ ist ein Induktorium, $f$ eine Funkenstrecke;

1) Siehe dazu: Nernst und v. Lerch, Annalen d. Physik, IV. Folge, Bd. 15 S. 836 (1904). 
daran ist in der üblichen Weise ein Schwingungskreis angehängt, bestehend aus der Selbstinduktion $s_{1}$ und der Kapazität $C$. Dem Schwingungskreis ist ein zweiter Kreis gegenübergestellt, welcher die Brückenanordnung repräsentiert. Er besteht aus der Spuhle $s_{2}$, dem Messtrog $t_{1}$ und dem Röhrehentrog $t_{2}$. Von der Mitte von $s_{2}$ führt eine Brücke zur Verbindung von $t_{1}$ mit $t_{2}$; in der Brücke liegt das Nullintrument $n$. Durch enge Koppelung zwischen $s_{1}$ und $s_{2}$ werden die Seh wingungen des ersten Kreises dem zweiten aufgezwungen und können durch entsprechende Änderung der Kapazitäten von $t_{1}$ und $t_{2}$ so verteilt werden, dass $n$ ein Minimum anzeigt.

Im speziellen ist der Apparat folgendermaassen gebaut: Das mittelgrosse Induktorium ist mit Deprez-Unterbrecher versehen und wird mit 12 Volt betrieben. Die Funkenstrecke besteht aus zwei Zinkstäben, deren Abstand zweckmässig durch eine Mikro-

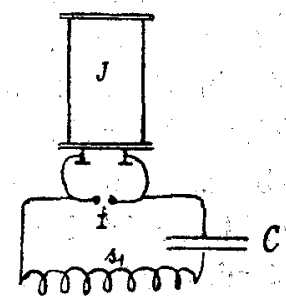
meterschraube geändert werden kann, weil auf die Weise leicht die Empfindlichkeit des später zu erwähnenden Nullinstrumentes zu ändern ist. Der $\mathrm{Kondensat} \mathrm{or} C$ ist eine $2 \mathrm{~mm}$ dicke Glasplatte, welche Stanniolbelegungen von der Grösse $15 \times 20 \mathrm{~cm}$ hat. Die Spule $s_{1}$ besteht aus 16 Windungen eines $0,8 \mathrm{~mm}$ starken Kupferdrahtes, welche in ca. $1 \mathrm{~cm}$ gegenseitigem $\mathrm{Ab}$ stand um einen gewöhnlichen Zylinder für Gasbeleuchtung herumgelegt sind. Diese Spule ist in die Spule $s_{2}$ hineingeschoben. $s_{2}$ besteht aus einem Glasrohr von $30 \mathrm{~cm}$ Länge und $8 \mathrm{~cm}$ Durehmesser; darum sind, eng gewickelt, 66 Windungen des $0,8 \mathrm{~mm}$-Drahtes herumgelegt; der Draht ist in der Mitte geteilt und die beiden Enden zu einer mit Hartgummi isolierten Klemmschraube geführt, ebenso geht der Draht an den beiden Enden der Spule zu isolierten Klemmschrauben. Der meist benutzte Röhrchentrog ist innen $6,5 \mathrm{~cm}$ hoch, $3,5 \mathrm{~cm}$ breit und $2 \mathrm{~cm}$ tief. Die in ihn in einem Abstand von ca. 1,5 cm eingêhängten Elektroden sind Silberbleche von der Grösse $5 \times 2,5 \mathrm{~cm}$. Zwischen die Elektroden ist oben ein Hartgummideckel eingeklemmt, der zur Aufnahme der Röhrchen durchbohrt ist. Meist wurden zwei Röhrchen eingesetzt; sie reichen bis zum Boden des Trogs, ihre Wandstärke beträgt. 
$0,3 \mathrm{~mm}$. Von der Weite der Röhrehen wird später die Rede sein. Der Trog wird mit Leitfähigkeitswasser (Kahlba u m) gefült; in die Röhrehen können Lösungen gefüllt werden. Die Dimensionen dieses Troges habe ich nach den Dimensionen des gleich zu beschreibenden Messtrogs eingerichtet; Fläche und Abstand der Elektroden wurden relativ klein gewählt, damit bei grösserer Elektrodentläche im Messtrog Kapazitätsänderungen in Röhrchentrog relativ grosse Elektrodenverschiebungen zur Kompensation nötig machten. Der Messtrog ist ein Glasrohr von $5 \mathrm{~cm}$ Durchmesser und ca. $10,5 \mathrm{~cm}$ Höhe. Seine Elektroden sind kreisförmige Scheiben aus Silberblech von 4,5 cm Durchmesser. Der zentral eingelötete Draht der unteren Elektrode ist ins Glasrohr unten eingeschmolzen, der obere Draht ist mit der Mikrometerschraube verbunden. Der Trog war gewöhnlich bis zur Hälfte mit Leitfähigkeitswasser gefüllt. Messtrog und Röhrchentrog sind gut gegen die Unterlage isoliert. Bei der Verbindung der einzelnen Bestandteile miteinander wurde für gute Kontakte gesorgt, und die Drähte wurden gestreckt geführt, um nicht durch variable Nebenkapazitäten und -selbstinduktionen Störungen in das System hineinzubringen.

Es erübrigt nun noch die Beschreibung des Nullinstrumentes. Als solches fanden anfangs ein Funkenindikator nach $\mathrm{Nernst}^{1}$ ) und ein elektrolytischer Detektor nach Nernst ${ }^{2}$ ) und Schloemilch ${ }^{3}$ ) Verwendung. $\mathrm{Zu}$ allen definitiven Messungen benutzte ich dann aber auf den Rat von Herrn Privatdozenten Dr. Zahn in Kiel einen Bleiglànzdetektor nach Braun, der sich für meine Zwecke den anderen Detektoren überlegen zeigte. Für diesen wie für manchen anderen Ratschlag bin ich Herrn Zahn zu grossem Dank verpflichtet. Bei dieser Gelegenheit sei es mir auch erlaubt, Herrn Geheimrat Dieteri ci dafür zu danken, dass er mich für die Fortführung meiner Untersuchung mit einigen Hülfsmitteln des Kieler physikalischen Instituts unterstützte. - Die Schaltung des Bleiglanzdetektors ist aus Fig. 6 ersichtlich. $s_{2}, t_{1}$ und $t_{2}$ sind die schon erwähnten Spulen und Tröge der Brückenanordnung. Die Brücke ist durch die kleine Spule $s_{3}$ gebildet; sie besteht aus neun Windungen eines $2 \mathrm{~mm}$ dicken Kupferdrahtes, die eng um ein $2,6 \mathrm{~cm}$ dickes Rohr gewickelt sind. Die

1) Nernst, Wiedemann's Annalen Bd. 60 S. 602 (1897).

2) Nernst und v. Lerch, l.c.

3) Schloemilch, Elektrotechn. Zeitschr. 1903 Nr. 47. 
Spannung, die gegebenenfalls in dieser Spule besteht, wird dann durch die in $s_{3}$ steckende Spule $s_{4}$, welche aus etwa 80 Windungen eines um ein Rohr von $1,9 \mathrm{~cm}$ Durchmesser herumgelegten $0,6 \mathrm{~mm}$ dicken Drahtes besteht, herauftransformiert. An $s_{4}$ ist der Detektor $d$ angelegt; er besteht aus einem Stückchen Bleiglanz, dessen einer glatt polierter Fläche eine Graphitspitze (Bleistift) leicht durch eine

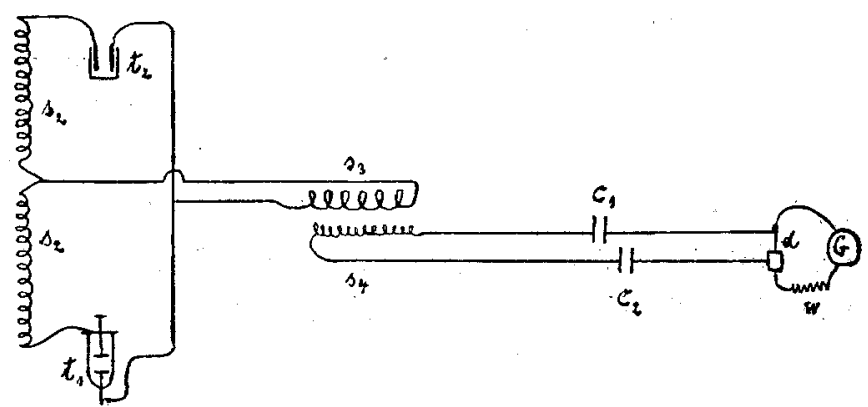

Fig. 6 .

Feder angedrückt ist. Dem Detektor parallel liegt ein Zeigergalvanometer von Hartmann \& Braun (G) mit vorgeschaltetem Widerstand $w$. Gehen Schwingungen durch den Detector, so wird er Sitz einer elektromotorischen Kraft, der resultierende Strom lenkt die Galvanometernadel ab. Damit der auftretende Strom sich nicht durch $s_{4}$ kurz schliesst, sind in die Leitung zwei Blockkondensatoren $C_{1}$ und $C_{2}$ von je zwei Mikrofarad eingeschaltet. Die Windungen von $s_{3}$ und $s_{4}$ sind in der Richtung der Kraftlinien von $s_{1}$ und $s_{2}$, d. h. senkrecht zu deren Windungen gelégt, um das Nullinstrument dem Wirkungsbereich von $s_{1}$ und $s_{2}$ zu entziehen; aus dem gleichen Grunde liegt der Detektor in ziemlicher Entfernung vom schwingenden System. Wird nun der Detektor als Nullinstrument benutzt, so hat man darauf zu achten, wann der Ausschlag der Galvanometernadel ein Minimum ist.

Die Genauigkeit der Messungen mit dem geschilderten Apparat hängt von verschiedenen Umständen ab, welche zum Teil später noch zu erörtern sind. Unter günstigen Bedingungen - $d . h$. in erster Linie: bei Füllung der Röhrchen mit Wasser oder mit einer starken Elektrolytlösung - beträgt sie $1-2 \%$. Diese nicht gerade grosse Genauigkeit ist wohl damit in Zusammenhang zu bringen, dass durch die Elektrodenverschiebung in Messtrog nicht bloss die Kapazität, sondern auch der $\mathrm{Oh} \mathrm{m}$ ' sche Widerstand geändert wird. 
Vorversuche: Wie gesagt, war es nötig, zuerst einmal festzustellen, wie weit die Kapazität des Röhrchentroges sich ändert, wenn die innere Leitfähigkeit der Röhrchen durch Beschicken mit verschieden starken Elektrolytlösungen geändert wird. Zu diesem Zweck wurden die Röhrchen nacheinander mit Leitfähigkeitswasser, 0,001-, 0,01-, 0,1und 1,0-norm.-KCl, gefüllt, und für jede Lösung wurde der Elektrodenabstand am Messtrog aufgesucht, bei dem die Galvanometernadel ein Minimum anzeigte. Die Röhrchen wurden stets einigermaassen genau bis zur Nivaugleichheit mit dem Leitfähigkeitswasser im Trog ausserhalb der Röhrchen gefüllt. (Das Niveau im Trog stand aber nicht in allen Versuchen gleich hoch.) - Es erwies sich ferner noch als notwendig, zu probieren, wie die Weite der Röhrchen, d. h. das Verhältnis der Elektrolytfüllung zur Menge des Wassers ausserhalb der Röhrchen, die Kapazität des Röhrchentroges beeinflusst.

Die Ergebnisse dieser Messungen sind in den Kurven der Fig. 7 zusammengefasst. Auf den Ordinaten sind die Abstände der beiden Elektroden im Messtrog verzeich-

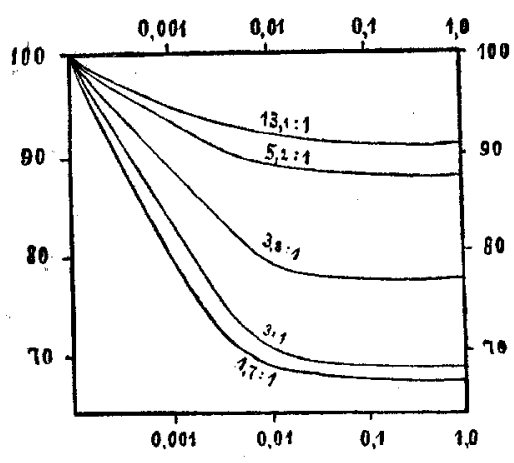

Fig. 7. net, der Abstand bei Füllung der Röhrchen mit Wasser gleich 100 gesetzt. Auf der Abszisse sind die Logarithmen der Elektrolytkonzentrationen des Röhreheninhaltes abgetragen. Das Zahlenverhältnis, das den einzelnen Kurven beigeschrieben ist, bedeutet das Verhältnis des Volumens von Wasser ausserhalb der Röhrchen zum Volumen der Lösung innerhalb der Röhrchen.

Man sieht, dass kleine Elektrolytkonzentrationen von grossem Einfluss auf die Kapazität sind, während weitere Konzentrationssteigerungen den Elektrodenabstand im Messtrog nur noch wenig zu verringern vermögen. Im speziellen fand ich, dass eine Steigerung der Konzentration von 0,1-norm. auf 1,0-norm. an der Minimumstellung des Nullinstrumentes gar nicht erkannt werden kann, und dass die Steigerung von 0,01 norm. auf 0,1 norm. zwar offenbar noch einen kleinen Unterschied im Elektrodenabstand ausmacht, dass sie aber im einzelnen Versuche bei der prozentischen Genauigkeit 
Eine Methode, die elektr. Leitfähigkeit im Innern von Zellen zu messen. $245^{5}$

meiner Versuchsanordnung nicht sicher festgestellt werden kann. Diese zunächst sehr unerfreuliche Erfahrung veranlasste mich, die Röhrchen versehiedener Weite durchzuprobieren, um die günstigste Weite herauszufinden. Die Kurven zeigen jedoch, dass eine Steigerung 'der Empfindlichkeit für das für die physiologischen Fragen gerade besonders kritische Gebiet von 0,01 bis 0,1 nicht zu erzielen war. Auch Versuche mit einem parallelepipedischen Trog ohne Röhrchen, der zur Stromzuführung aussen auf zwei gegenüberliegenden Flächen mit Stanniol belegt war und ganz mit den verschiedenen Elektrolytlösungen gefüllt wurde, lieferten keine günstigeren Resultate.

Es ist also nicht möglich, am Elektrodenabstand zu erkennen, ob die innere Leitfähigkeit einer 0,01- oder einer 0,1-normal-Salzlösung entspricht. Die Durchführung des Versuchsplanes würde damit vereitelt gewesen sein, wenn sich nicht ein anderes Mittel zur Unterscheidung der stärkeren Elektrolytkonzentrationen geboten hätte. Alle Messungen wurden so ausgeführt, dass die verschiebliche Elektrode des Messtrogs 2-3 mal von oben und 2-3 mal von unten her in die Minimumstellung gebracht wurde. Dabei ergab sich, dass die Breite des Minimums stark variiert, und dass diese Variationen sich auch auf das Gebiet zwischen 0,01-norm. und 0,1-norm. erstrecken. Ein weiteres Unterscheidungsmerkmal für die verschiedenen Konzentrationen ist die Geschwindigkeit, mit welcher die Galvanometernadel in die Nullstellung einrückt. Genauer ausgemessen wurde in den bisherigen Versuchen nur die Breite des Minimums.

In der folgenden Tabelle I gebe ich zwei Versuchsbeispiele:

Tabelle I.

\begin{tabular}{l|c|c|c|c}
\hline $\begin{array}{c}\text { Konzentration } \\
\text { in den } \\
\text { Röhrchen }\end{array}$ & $\begin{array}{c}\text { Elektroden- } \\
\text { abstand (für } \\
\text { Wasser=100) }\end{array}$ & $\begin{array}{c}\text { Minimum- } \\
\text { breite in } \\
\text { mm }\end{array}$ & $\begin{array}{c}\text { Elektroden- } \\
\text { abstand (für } \\
\text { Wasser=100) }\end{array}$ & $\begin{array}{c}\text { Minimum- } \\
\text { breite in } \\
\text { mm }\end{array}$ \\
\hline & 86,7 & $\mathbf{4 , 1}$ & 84,2 & $\mathbf{2 , 5}$ \\
0,001 -norm. & 79,0 & $\mathbf{3 , 0}$ & $\mathbf{7 5 , 5}$ & $\mathbf{2 , 7}$ \\
$\mathbf{0 , 0 0 2} "$ & 69,7 & $\mathbf{1 , 8}$ & 67,6 & $\mathbf{1 , 4}$ \\
$0,005 \%$ & 66,9 & $\mathbf{1 , 4}$ & 65,9 & $\mathbf{1 , 4}$ \\
$\mathbf{0 , 0 1} "$, & 67,4 & $\mathbf{0 , 7}$ & $\mathbf{6 6 , 0}$ & $\mathbf{0 , 5}$
\end{tabular}

Man sieht, wie sich 0,01-norm. und 0,1-norm. in der Breite des Minimums deutlich voneinander unterscheiden. Auch der Gang der Galvanometernadel ist im ersten Fall erheblich langsamer als im zweiten. 
Die Erklärung für diese Erscheinungen ist wohl darin zu suchen, dasss bei geringen Leitfähigkeiten in den Röhrchen Energie absorbiert wird:. Bei Füllung der Röhrehen mit Wasser ist das Minimum scharf, weil die Trogfüllung für die schnellen Sehwingungen als guter Isolator fungiert, in dem bei der dielektrischen Leitung keine Energie absorbiert wird. Bei Füllung mit einer gut leitenden Lösung findet ebenfalls kein oder fast kein Energieverbrauch statt, weil der Widerstand zu gering ist. Absorption von Energie findet erst statt, wenn die Leitfähigkeit relativ klein ist, und dann wird zugleich das Minimum unscharf, weil eigentlich zwei Minima vorhanden sind, ein Kapazitäts- und ein Widerstandsminimum, welche nicht zusammenfallen. Man körnte das Minimum wieder schärfer machen, wenn man dem Messtrog einen entsprechenden Widerstand parallel schaltete.

Jedenfalls ist es nach diesen Versuchen möglich, grössere innere Leitfäbigkeiten an der Breite des Minimums, kleinere am Elektrodenabstand des Messtrogs zu erkennen.

Die innere Leitfähigkeit der Blutkörperchen: Bisher erstrecken sich meine Untersuchungen allein auf Blutkörperchen. Diese wurden aus dem defibrinierten Blut vom Rind, Kalb oder Schwein durch Zentrifugieren mit 7\% iger Rohrzuckerlösung gewonnen. Der Rohrzucker (Saccharose, Ka hl b a u m) war in Leitfähigkeitswasser gelöst. In den meisten Versuchen wurden die aus dem Serum ausgeschleuderten Blutkörperchen 4 mal je 4 Minuten in einer Runneschen Zentrifuge bei ca. 3500 Umdrehungen mit reichlicher Rohrzuckerlösung zentrifugiert. Saugt man jedesmal die überstehende Lösung mit einer Kapillare ab,' so erhält man dann einen Brei, dessen Kohlrausch'sche Leitfähigkeit zwischen der einer 1/2000und einer $1 / 4000$-norm.-KCl-Lösung liegt. Wenn auch bei dieser Prozedur, wie aus den Versuchen von Gürber ${ }^{1}$ ) hervorgeht, Elektrolyt aus den Blutkörperchen austritt, so geht aus allem Weiteren hervor, dass jedenfalls die meisten Salze im Innern bleiben.

Bei der geringen Kohlrausch'schen Leitfähigkeit der Blutkörperchen konnten nun die Versuche zur Messung ihrer in neren Leitfähigkeit einfach so vorgenommen werden, dass die Blutkö rperchen in die Röbrchen des Röhrchentrogs gefüllt wurden; es musste sich

1) Gürber, Salze des Blutes. II. Würzburg 1904. 
Eine Methode, die elektr. Leitfähigkeit im Innern von Zellen zu messen. $247^{3}$

dann ja zeigen, ob ihre innere Leitfähigkeit grösser als ihre Kohlrausch'sche Leitfähigkeit ist, d. h. ob sie die Kapazität des Troges mehr steigern, als wenn die Röhrehen mit einer 1/2000- bis 1/4000-norm.-KCl-Lösung gefüllt sind.

Die zwei Röhrchen des Troges hatten in allen diesen Versuchen einen inneren Durchmesser von ca. $11 \mathrm{~mm}$.

Ich teile nun zunächst eine Anzahl von Versuchen mit:

\section{Tabelle II.}

1. Blut vom Schwein. Leitfähigkeit $=$ ca. 1/2200-norm, $-\mathrm{KCl}$.

$\begin{array}{lcrc} & \begin{array}{r}\text { Elektrodenabstand } \\ \text { (für Wasser }=100)\end{array} & \text { Elektrodenabstand } \\ 0,001 \text {-norm.-KCl } & 80,1 & \text { Blutkörperchen } & 74,6 \\ 0,01 . " & 69,4 & & \\ 0,1 \quad \cdots & 68,7 & \end{array}$

2. Blut vom Schwein. Leitfähigkeit $=$ ca. ${ }^{1 / 2600}$-norm:-KCl.

$\begin{array}{llcr}0,001-\text {-norm.-KCl } & 79,6 & \text { Blutkörperchen } \\ 0,002 \% & 74,4 & & 65,9 \\ 0,005 & & 60,8 & \\ 0,01 \quad \# & 61,1 & \\ 0,1 & & \end{array}$

3. Blut vom Rind. Leitfähigkeit $=$ ca. 1/2400-norm.-KCl.

$\begin{array}{lcc}0,002-\text { norm.-KCl } & 74,2 & \text { Blutkörperchen } \\ 0,005 \quad \# & 66,4 & 69,4 \\ & & \end{array}$

4. Blut vom Rind. Leitfähigkeit $=$ ca. 1/2300-norm.-KCl.

\begin{tabular}{|c|c|c|c|c|c|}
\hline $0,001-$ & $\mathrm{m} \cdot-\mathrm{KCl}$ & 86,7 & Blutkörperchen & & 70,5 \\
\hline 0,002 & $\eta$ & 79,0 & $\therefore$ & + Saponin & 66,1 \\
\hline 0,005 & $n$ & 69,7 & & & \\
\hline 0,01 & $\eta$ & 66,5 & 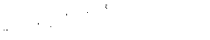 & & \\
\hline 0,1 & $"$ & 67,2 & & & \\
\hline & 5. B & Kalb. & gkeit $=$ ca. $1 / 34$ & 00-norm.-KCl & \\
\hline $0,001-$ & $\mathrm{m} \cdot-\mathrm{KCl}$ & 84,2 & Blutkörperchen & & 70,6 \\
\hline 0,002 & $\eta$ & 75,5 & $n$ & + Saponin & 68,3 \\
\hline 0,005 & $n$ & 67,6 & & & \\
\hline 0,01 & $n$ & 65,4 & & & \\
\hline 0,1 & " & 65,9 & & & \\
\hline
\end{tabular}

Vergleicht man den Einfluss der KCl-Lösungen auf die Kapazität in diesen Versuchen mit dem Einfluss der Blutkörperchen, so wird man finden, dass die Blutkörperchen etwa so wirken wie eine KCl-Lösung von $0,002-0,005$-Normalität. Man wird daraus zunächst den Schluss ziehen, dass im Innern der Blutkörperchen nur selır wenig freie Elektrolyte vorhanden sind, dass die meisten der Salze, die sich in der Asche der Blutkörperchen finden, organisch 
gebunden sind. Zu diesem Schluss passt dann auch gut, dass, wenn man die Blutkörperchen mit etwas Saponin zerstört - in jedes Röhrchen wurde ein Tropfen einer mässig starken, wässerigen Saponinlösung gegeben -, die innere Leitfähigkeit der Röhrchen, wie die Tabelle zeigt, ansteigt. Es sieht ganz so aus, als ob durch das Abtöten der Blutkörperchen die vorher gebundenen Salze frei werden; auch die Kohlrausch'sche Leitfähigkeit der Blutkörperchen steigt ja stark, wenn man mit Saponin hämolysiert, und auch diese Steigerung kann auf Lösung der Salze aus einer natürlichen organischen Bindung bezogen werden.

Der Einfluss des Blutkörperchen-Gresamtvolumens: Es ist aber ein Bedenken geltend zu machen. Wenn man sich in der beschriebenen Weise einen Blutkörperchenbrei herstellt, so ist nicht daran zu denken, dass man nun 100 Volumprozent Blutkörperchen in dem Brei hat, sondern sicherlich ist noch etwas Zwischenflüssigkeit da, welche die Blutkörperchen voneinander trennt; die Blutkörperchen "sind durch eine dielektrische Hülle, dargestellt durch etwas Rohrzuckerlösung, gesondert. Es fragt sich nun, ob das nicht auf dasselbe hinauskommt, als wenn gan z mit Blutkörperchen ausgefüllte, aber engere Röhrchen als in Wirklichkeit in dem Trog steckten. Und die Kurven der Fig. 7 (S. 244) lehren ja, wie die Kapazität von der Weite der Röhrehen abhängig ist. In der Tat ist denn auch leicht nachzuweisen, dass das BlutkörperchenGesamtrolumen von grossem Einfluss ist, wie die Tabelle III zeigt:

Tabelle III.

1. Blut vom Kalb.

Blutkörperchen : . . . . . . . . .

1 Teil Blutkörperchen +1 Teil Rohrzuckerlösung

$\begin{array}{cc}\begin{array}{c}\text { Elektroden- } \\ \text { abstand }\end{array} & \begin{array}{c}\text { nach } \\ \text { Saponin }\end{array} \\ 71,0 & 66,15 \\ 80,0 & 66,3\end{array}$

2. Blut vom Kalb.

1 Teil Blutkörperehen +1 Teil Rohrzuckerlösung

$1 \% "+3$

$\begin{array}{ll}69,1 & - \\ 76,3 & - \\ 79,5 & 67,6\end{array}$

3. Blut vom Rind.

Blutkörperchen *. . . . . . . . . .

1 Teil Blutkörperchen +1 Teil Rohrzuckerlösung

1 $+3$

$\begin{array}{cc}70,6 & 68,3 \\ 79,0 & - \\ 86,9 & 66,3\end{array}$

$\because$ Je geringer also das Blutkörperchenvolumen, um so geringer die Kapazitätserhöhung. Dabei sind an und für sich genug Elektrolyte 
Eine Methode, die elektr. Leitfähigkeit im Innern von Zellen zu messen. 249

auch in 25 Volumprozent Blutkörperchen vorhanden, um, durch Saponin frei gemacht bzw. gleichmässig in den Röhrehen verteilt, die Kapazität gerade so stark zu erhöhen wie der unverdünnte Blutkörperbrei nach Saponinbehandlung; auch das lehrt die Tabelle.

Auf jeden Fall folgt aus diesen Versuchen, dass die bisher mitgeteilten Werte kein reiner Ausdruck der inneren Leitfähigkeit der Blutkörperehen sein können, sondern dass man sich zunächst erst fragen muss, wie gross die Kapazitätssteigerung bei 100 Volumprozent Blutkörperchen ist.

Der Einfluss stärkeren Zentrifugierens: Um diese Frage zu beantworten, wurden die Blutkörperchen mit Hilfe einer Thilenius-Zentrifuge ${ }^{1}$ ) noch stärker zusammengepresst, und die überstehende Lösung wurde mit besonderer Vorsicht durch eine Kapillare abgesaugt. Der Effekt ist deutlich:

\section{Tabelle IV.}

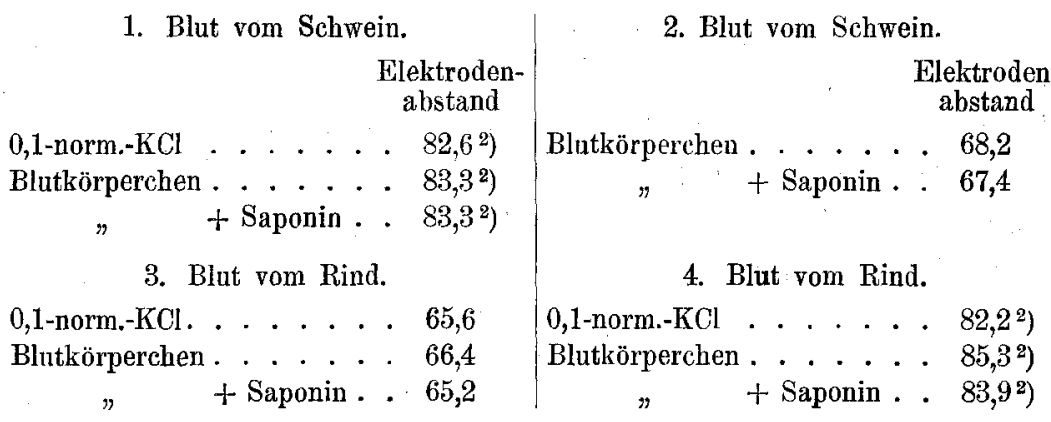

Es ist ersichtlich, dass nun nicht mehr der Elektrodenabstand dem einer $0,002-0,005$-norm-KCl.-Lösung entspricht, wie in den ersten Versuchen (Tabelle II), sondern dass der Elektrodenabstand durch das starke Zentrifugieren fast auf sein Minimum, wie es einer 0,1-norm.-KCl-Lösung entspricht, herabgedrückt ist. Dazu stimmt, dass der Einfluss des Saponins auf den Elektrodenabstand fast verschwunden ist. In der folgenden Tabelle stelle ich die Differenzen des Elektrodenabstandes vorund nach Saponinbehandlung aus den Tabellen II, III und IV zusammen, als Ausdruck dafür, wie das Blutkörperchen-Gesamtvolumen dièse Werte beeinflusst:

1) Siehe Koeppe, Pflüger's Arch. Bd. 107 S. 183 (1905).

2) Nur ein Röhrchen war mit Blutkörperchen bzw. Lösung gefüllt, das andere mit Wasser. 


$\begin{array}{ccc}\text { Kleines } & \text { Tabelle V. } & \\ \begin{array}{c}\text { Grosses } \\ \text { Blutkörperchenvolumen } \\ \text { (Tab. III) }\end{array} & \begin{array}{c}\text { Fast 100 Volum- } \\ \text { Blutkörperchenvolumen } \\ \text { (Tab. III) }\end{array} & \begin{array}{c}\text { prozente Blutkörperchen } \\ \text { (Tab. IV) }\end{array} \\ 11,9 & 5,4 & 0 \\ 20,6 & 4,5 & 0,8 \\ - & 4,4 & 1,2 \\ - & 2,3 & 1,4\end{array}$

Danach darf man wohl behaupten, dass, wenn es gelänge, die Blutkörperchen in grösserer Menge vollkommen von anhaftender Lösung zu trennen, sie die Kapazität gerade so steigern würden wie die stärkste der Salzlösungen, gleichgültig ob sie hämolysiert wären oder nicht.

Danach ist es aber auch als erwiesen anzusehen, dass die innere Leitfähigkeit der Blutkörperchen mindestens der einer 0,01-norm.-Salzlösung gleichkommt, vielleicht erheblich grösser ist, da 0,01-, 0,1- und 1,0-norm.-Lösungen auf den Elektrodenabstand gleich wirken.

Welche dieser Leitfähigkeiten wirklich vorhanden ist, lässt sich nun, wie wir (S. 245) sahen, eventuell herausbekommen, wenn man bei der Messung die Breite des Minimums beachtet.

Die Breite des Minimums: In einer Anzahl von Versuchen wurde die Breite des Minimums möglichst sorgfältig bestimmt; die Ergebnisse enthält die folgende Tabelle:

\section{Tabelle VI.}

\section{Breite}

1. $7 \%$ ige Rohrzuckerlösung $0,4 \mathrm{~mm}$ Blutkörperchen . . . . 0,1 " 0,001 -norm.-KCl . . . 3,2 " 0,1-norm.KCl . . . . 0,4"

3. $7 \%$ ige Rohrzuckerlösung $1,1 \mathrm{~mm}$ Blutkörperchen . . . . 1,1" $\begin{array}{ccc}n & + \text { Saponin } 1,0 \% \\ 0,1 \text { norm.-KCl } & \text {. . . . } 0,6 "\end{array}$

5. $7 \%$ ige Rohrzuckeriösung $0,9 \mathrm{~mm}$ Blutkörperchenn .. . . . . 1,1 " 0,01-norm.-KCl . . . 1,6 " 0,1 -norm.-KCl . . . 0,6 "
2. $7 \%$ ige Rohrzuckerlösung $0,4 \mathrm{~mm}$ Blutkörperchen . . . . 0,8 "

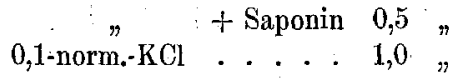

4. $7 \%$ ige Rohrzuckerlösung $1,0 \mathrm{~mm}$ Blutkörperchen . . . . . 0,6" 0,01-norm.-KCl . . . 1,5 " 0,1-norm.-KCl. . . . . 0,5 "

6. Blutkörperchen . . . . $0,8 \mathrm{~mm}$ 0,005-norm.-KCl . . . 4 4,9 " 0,01-norm.-KCl . . . . 1,2" 0,1-norm.-KCl . . . . 0,6 " Breite

7. Blutkörperchen . $0,6 \mathrm{~mm}$ 0,01-norm.-KCl . 1,6 " 0,1 -norm.- $\mathrm{KCl}$. $0,6 \%$ 
Man sieht, dass das Minimum bei den Blutkörperchen oft so scharf ist, wie es nur sein kann, und dass es im allgemeinen an Schärfe kaum dem Minimum bei einer 0,1 norm.-KCl-Lösung nachsteht. Was aber wichtiger ist, ist das, dass das Minimum bei den Blutkörperchen stets erheblich schärfer ist als bei einer 0,01-norm.KCl-Lösung. Daraus folgt, dass die innere Leitfähigkeit der Blutkörperchen annähernd gleich der einer 0,1-norm.Salzlösung ist.

Dies ist das Ergebnis meiner Untersuchungen. Es gelingt also, das Innere von Zellen zu analysieren, ohne die Zellen zu verletzen. Was hier an Blutkörperchen gemacht ist, wird sich natürlich auch an anderen Zellen und Geweben ausführen lassen. Der Grad der Genauigkeit der Analyse ist freilich bisher kein sehr grosser, dadurch, dass in unvorhergesehener Weise gerade in dem für die physiologischen Fragen kritischen Konzentrationsgebiet die Methode an Leistungsfähigkeit einbüsst. Ich hoffe, dass eine andere Methode zur Messung der inneren Leitfähigkeit, die demnächst probiert werden soll, noch mehr leistet.

Schlussfolgerungen: Es sei nun noch einiges darüber gesagt, inwiefern die Versuche über ihr faktisches Ergebnis hinaus eine Bedeutung haben. Da ist in erster Linie an die altbekannte Tatsache zu erinnern, dass die Blutkörperchen, wie sonst noch viele Zellen, andere Salze oder wenigstens die Salze in anderer Zusammensetzung enthalten als ihre Umgebung. So oft diese Tatsache konstatiert wurde, war sie gleichbedeutend mit der Frage, wie die Differenzen zu erklären sind. Und da können hauptsächlich zwei Deutungen gegeben werden; entweder führt man die Differenzen darauf zurück, dass die Salze aussen und innen zwar in freiem Diffusionsaustausch miteinander stehen, dass aber ein Teil von ihnen durch Bindung an organisehe Komponenten des Zellinhalts festgebalten und angehäuft wird, während der Inhalt zu einem anderen Teil der Salze keine Affinität äussert. Oder man nimmt an, dass die Zelloberfläche, die Plasmahaut, für die Salze ein Diffusionshindernis bedeutet, vermöge dessen bestehende Konzentrationsdifferenzen zwischen den freien Elektrolyten aussen und dẹnen innen aufrechterhalten werden. Schliesst man sich der zweiten Deutung an, so ist die Konsequenz, dass man die Zelloberfläche mit der Fähigkeit begabt denken muss, Stoffe, welche nicht in die Zellen hineindiffundieren können, eventuell von sich aus aktiv hinein- 
zubefördern; eine andere Konsequenz ist die, dass, wenn gewisse Salze die Funktion von Zellen zu beeinträchtigen vermögen, man diese giftige Wirkung an eine Veränderung der bei der Funktion beteiligten Oberfläche gebunden zu denken bat. Genug, man hat der Plasmahaut komplizierte Leistungen zuzutrauen. Man ist also je nach dem Bild, das man sich von dem Zustand der Salze in den Zellen macht, genötigt, zu wichtigen Fragen des Zellebens eine ganz verschiedene Stellung einzunehmen.

Ich selbst habe mehrfach die zweite der genannten Auffassungen vertreten, aus welchen Gründen, soll hier nicht wiederholt werden, ebensowenig, warum ich die Gegengründe, welche von J. Loeb, Bang, Moore und Roaf u. a. vorgebracht worden sind, nicht für überzeugend halten $\operatorname{kann}^{1}$ ). Die hier mitgeteilten Versuche bestärken mich aber in meiner Ansicht, da hier der Nachweis geführt wird, dass wenigstens in den Blutkörperchen die Flektrolyte zum mindesten grösstenteils als frei gelöst anzusehen sind. - Freilich, der ganze Aufbau der Blutkörperchen bleibt auch weiter noch für uns recht rätselhaft; ich erinnere allein daran, dass noch jede befriedigende Erklärung für die so interessanten Angaben von Stewart²) fehlt, nach denen, je nach der Art des Eingriffs, von den lädierten Blutkörperchen bald das Hämoglobin; bald die Salze vorwiegend losgelassen werden. Einen neuen Einblick in die Struktur der Blutkörperchen scheinen mir die mitgeteilten Versuche höchstens noch insofern zu gewähren, als sie für eine gleichmässige Verteilung der Salze über den ganzen Blutkörpercheninhalt sprechen; denn bestände das Innere der Blutkörperchen zu einem erheblichen Teile aus einer "Gerüstsubstanz", welche keine Salze enthält, so könnte nach meinen Versuchen über den Einfluss des Blutkörperchen-Gesamtvolumens auf die innere Leitfähigkeit, d. h. nach dem Einfluss der Zwischen-

1) Siehe hierzu: Höber, Errgebnisse der wissenschaftl. Medizin Bd. 1 S. 119 (1910) und Biochem. Zeitschr. Bd. 20 S. 56 (1909). Auch das neueste, vor kurzem von Osterhout [Zeitschr. f. physik. Chemie Bd. 70 S. 408 (1910)] vorgebrachte Argument, dass Ca-Salze nachweislich in lebende Pflanzenzellen eindringen, kann ich für keinen Beweis der freien Diffusibilität durch die Plasmahaut hindurch ansehen; siehe dazu meine Ausführungen über physikalische und physiologische Permeabilität (Biochem. Zeitschr., l. c., und Physik. Chemie der Zelle und der Gewebe 1906 S. 178 ff.).

2) Stewart, Journ. of physiol. vol. 24 p. 211 (1899). 
Eine Methode, die elektr. Leitfähigkeit im Innern von Zellen zu messen. 253

schaltung nicht leitender Schichten zwischen leitende (S. 248) die innere Leitfähigkeit nicht so hoch gefunden werden, wie sie tatsächlich gefunden wurde.

\section{Zusammenfassung.}

Es wird eine Methode beschrieben, mit welcher es gelingt, die „innere Leitfähigkeit" von Zellen, d. h. die Leitfähigkeit des Inhalts der unverletzten Zellen zu messen. Es wird gefunden, dass Blutkörperchen, deren Leitfähigkeit, nach der Kohlrausch'schen Methode gemessen, fast gleich Null ist, eine innere Leitfühigkeit besitzen, welche ungefähr derjenigen einer 0,1-norm.-KCl-Lösung entspricht. Daraus ist zu schliessen, dass die Salze im Innern der Blutkörperchen, mindestens vorwiegend, frei und nicht organisch gebunden vorhanden sind. 